\title{
Synthesis of Mono-, Di- and Tricyclopentadienylphenylmethanes from Cyclopentadienyl(tributylphosphine)copper(I) and
}

\section{Bromophenylmethanes}

\author{
JAN-ERIK MÅNSSON and OLOF WENNERSTRÖM
}

Department of Organic Chemistry, Chalmers University of Technology and University of Göteborg, S-402 20 Göteborg, Sweden

Mono-, di- and tricyclopentadienylphenylmethanes have been prepared from cy. clopentadienyl(tributylphosphine)copper(I) and mono-, di- and tribromophenylmethane, respectively. Variable amounts of stilbenes were formed in the latter reactions. The thermal isomerisation of cyclopentadienyltriphenylmethane and dicyclopentadienyldiphenylmethane via $[1,5]$ sigmatropic hydrogen shifts has been studied by ${ }^{1} \mathrm{H}$ NMR spectroscopy. Some mechanisms of the cyclopentadienylcopper reactions are briefly discussed.

We have previously investigated the monomeric complex cyclopentadienyl(tributylphosphine)copper (I), $\left(\mathrm{CpCuPBu}_{3}\right)^{1}$ as a selective reagent for the conversion of organic halides to cyclopentadienyl compounds. Generally, alkyl- or arylcyclopentadienes are obtained from the corresponding halide and sodium or lithium cyclopentadienide or cyclopentadienylmagnesium bromide.2 However, these cyclopentadienides are strong nucleophiles and bases prone to react at electron-deficient sites or with acidic hydrogens. In contrast to the alkaline and alkaline-earth cyclopentadienides, $\mathrm{CpCuPBu}_{3}$ does not usually react with carbonyl groups or acidic hydrogens.

The reactions of $\mathrm{CpCuPBu}$ with iodoarenes, acyl chlorides ${ }^{4}$ and $\alpha$-halocarbonyl compounds ${ }^{5}$ as well as its reactions with chlorotriphenylmethane and dibromodiphenylmethane ${ }^{5}$ have been reported previously. The latter is the first reaction reported to give a dicyclopentadienyl

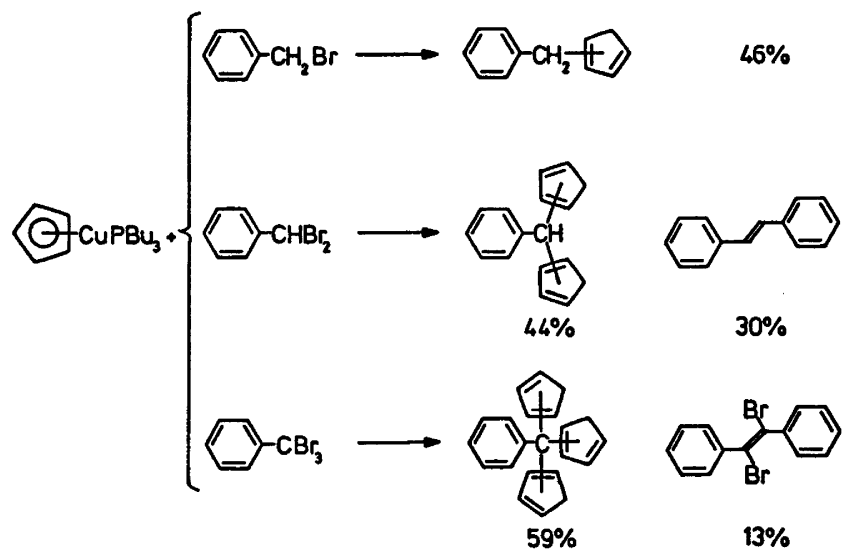

Scheme 1.

Acta Chem. Scand. B 32 (1978) No. 7 
compound from a cyclopentadienyl metal com. pound and an organic dihalide. We now report an extended investigation of the reactions between $\mathrm{CpCuPBu}_{3}$ and benzylic halides for the synthesis of di- and tricyclopentadienylphenylmethanes.

\section{RESULTS AND DISCUSSION}

Cyclopentadienyl(tributylphosphine)copper(I), ( $\left.\mathrm{CpCuPBu}_{3}\right)$, reacts with bromophenylmethane to give cyclopentadienylphenylmethane $(46 \%)$ and with bromodiphenylmethane to give cyclopentadienyldiphenylmethane. Dibromophenylmethane and tribromodiphenylmethane give dicyclopentadienylphenylmethane $(44 \%)$ and tricyclopentadienylphenylmeth. ane $(59 \%)$, respectively. In the latter reactions, $t_{\text {rans-stilbene }}(30 \%)$ and dibromostilbene (13\%), respectively, were formed (Scheme 1).

Isomerisations of cyclopentadienylmethanes. Monosubstituted cyclopentadienes are usually obtained as a mixture of isomers due to rapid thermal or base-induced isomerisation of the initially-formed 5-isomer to the more stable 1 and 2-isomers unless special precautions are made ' (Scheme 2). However, we observed

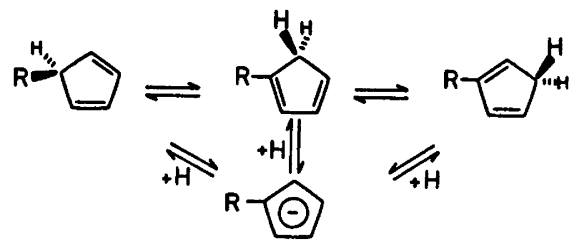

Scheme 2. repeatedly that $\mathrm{CpCuPBu}_{3}$ and chlorotriphenylmethane gave one single crystalline isomer, which was isolated by filtration after a few hours. The NMR spectrum showed, apart from the aromatic protons, three multiplets at $\delta 6.42,6.33$ and 6.28 and one multiplet at $\delta 2.89$ with the relative area 1:1:1:2. The NMR spectrum of the solution slowly changed to that of the equilibrium mixture reported earlier. We assume that pure 1-(triphenylmethyl)cyclopentadiene is formed by a rapid $[1,5]$ sigmatropic hydrogen shift from an initiallyformed 5-isomer. The rearrangement to the equilibrium mixture is a much slower process. If this assumption is correct we can assign the composition of the equilibrium mixture as $21 \%$ of the 1 -isomer and $79 \%$ of the 2-isomer. Similar results were obtained with cyclopentadienylphenylmethane, dicyclopentadienyldiphenylmethane and tricyclopentadienylphenylmethane. Rapid chromatography of the reaction products gave mixtures of isomers with a large excess of the 1-isomer which, on standing or treatment with base, rearranged to the equilibrium mixture containing more of the 2-isomers (Table 1).

Steric effects in cyclopentadienylphenylmethanes. In a recent series of papers, Mislow and co-workers have discussed the various conformations and dynamic processes in triaryland tetraarylmethanes. ${ }^{8}$ The exchange of a benzene ring for a cyclopentadienyl ring should not greatly affect the overall structure of the arylmethane and may rather be regarded as a small perturbation of the system. The cy-

Table 1. The ratio of 1-substituted to 2 -substituted cyclopentadienyl rings in some cyclopentadienylphenylmethanes.

\begin{tabular}{lll}
\hline Compound & \multicolumn{2}{c}{ Ratio of 1-substituted rings to 2-substituted } \\
\cline { 2 - 3 } & After isolation & At equilibrium \\
\hline Cyclopentadienylphenylmethane & $3.4: 1.0$ & $1.0: 1.0$ \\
Dicyclopentadienylphenylmethane & - & $1.2: 1.0$ \\
Cyclopentadienyldiphenylmethane & $1.8: 1.0$ & $1.2: 1.0$ \\
Cyclopentadienyltriphenylmethane & $a$ & $1.0: 3.8$ \\
Dicyclopentadienyldiphenylmethane & $3.0: 1.0$ & $1.0: 3.1$ \\
Tricyclopentadienylphenylmethane & $1.2: 1.0$ & $1.0: 3.8$ \\
\hline
\end{tabular}

${ }^{a}$ Only 1-(triphenylmethyl)cyclopentadiene. 
clopentadienyl ring is used as an interent probe to measure steric effects. The equilibrium ratio between the 1- and 2-isomers of strained cyclopentadienes can be compared with that of unstrained derivatives with electronically similar substituents. Assuming that the methylene group in the 1-isomers, but not in the 2isomers, is affected by the neighbouring groups

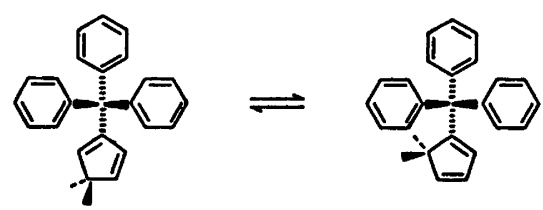

Scheme 3.

(Scheme 3), one can estimate for the 1-isomers the steric strain difference between the different compounds by simple calculation. With this assumption it is possible to compare small strain effects in diaryl-, triaryl- and tetraarylmethanes. The equilibrium ratios of 1 - and 2 -isomers of some cyclopentadienylphenylmethanes are collected in Table 1 together with the same ratios in the initially isolated products. In the series of tetrasubstituted methanes, the calculated energy difference between the isomers ranges from 2.8 to $3.3 \mathrm{~kJ} / \mathrm{mol}$ with an estimated error of $\pm 0.2 \mathrm{~kJ} / \mathrm{mol}$. In the di- and trisubstituted methanes little steric strain is present. The ratio of 1 - and 2-isomers is approximately the same as in methylcyclopentadiene and corresponds to an energy difference of up to $0.5 \mathrm{~kJ} / \mathrm{mol}$ in favour of the 1 -isomers. Thus the steric perturbation in 1-(triphenylmethyl)eyclopentadiene can be estimated to be $3.8 \mathrm{~kJ} / \mathrm{mol}$.

The mechanism of the reaction. $\mathrm{CpCuPBu}_{3}$ reacts like other organocopper compounds with a variety of organic halides to give new carbon carbon bonds. It reacts with $(-) \alpha$-bromophenylacetic acid to give racemic cyclopentadienylphenylacetic acid. ${ }^{\circ}$ We have also found that $\mathrm{CpCuPBu}_{3}$ reacts with para-substituted benzoyl chlorides in an $\mathrm{S}_{\mathrm{N}}$ 2-like reaction. ${ }^{10}$ The question is whether the cyclopentadienyl group or the copper atom is the nucleophile. In the former case one would expect $\mathrm{CpCuPBu}_{3}$ to be a stronger base, i.e. to react with water and other weak acids and to form a fulvene in the reaction with dibromophenylmethane, but this is not observed. If the copper atom acts as a nucleophile, the first step in the reaction would be the oxidative addition of a monohapto-CpCuPBu${ }_{8}$ to the carbon-halide bond. This addition should be of a polar type to be consistent with previous findings (Scheme 4).

In most reactions between $\mathrm{CpCuPBu}_{3}$ and bromophenylmethanes by-products, arising from reductive dimerisation of the bromophenylmethanes, were isolated. An alternative mechanism, successive one-electron transfers from $\mathrm{CpCuPBu}_{3}$ to the substrate followed by radical couplings, seems to operate in these reactions.

$D i$ - and tricyclopentadienylphenylmethanes as quinacene percursors. The synthesis of con. densed cyclopentadienes, polyquinanes, with the ultimate goal of preparing dodecahedrane attracts many organic chemists. ${ }^{11}$ The compound $\mathrm{C}_{16}$-hexaquinacene has recently been synthesised by a multistep sequence. ${ }^{12}$ An alternative synthetic approach is afforded by an intramolecular cyclisation of tricyclopentadienylphenylmethane. There are six double bonds available for ring-closure reactions. The $\left[4_{s}+4_{s}+4_{s}\right]$ cyclisation of tri(5-cyclopentadienyl)phenylmethane, which is a very unlikely isomer, is photochemically allowed in a concerted reaction (Scheme 5). However, other

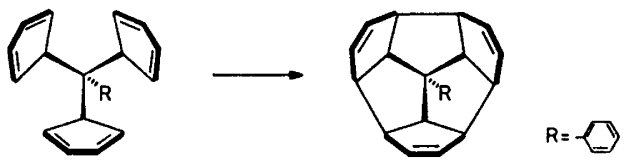

Scheme 5.

energetically favourable reactions of the more stable isomers may well lead to hexaquinacenetriene.

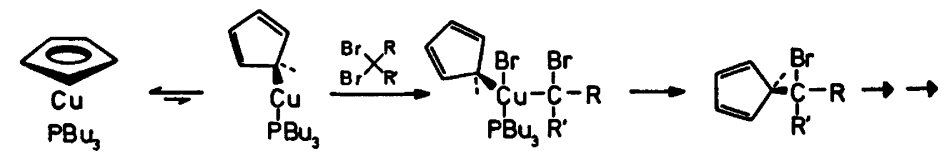

Scheme 4.

Acta Chem. Scand. B 32 (1978) No. 7 


\section{EXPERIMENTAL}

UV and IR spectra were recorded on a Beckman DK-2A or an IR-10. NMR spectra were recorded either on a Varian A-60 or a Bruker WH 270 instrument with $\mathrm{CDCl}_{3}$ as solvent. Mass spectra were recorded on an AEI MS 902 instrument. $\mathrm{CpCuPBu}_{3}$ was prepared by the method given by Cotton and Marks. ${ }^{13}$ All reactions with $\mathrm{CpCuPBu}_{3}$ were run in dried apparatus under dry, oxygen-free nitrogen.

General procedure for the reaction of $C p C u P B u_{3}$ and benzylic halides. An ethereal solution of the halide was added dropwise to a freshly prepared solution of $\mathrm{CpCuPBu},(50 \%$ excess $)$ at $0^{\circ} \mathrm{C}$. The mixture slowly turned yellow, and frequently a slight clouding appeared. Stirring was continued overnight while the mixture reached room temperature. A small amount of $\mathrm{CCl}_{4}$ was added to destroy excess $\mathrm{CpCuPBu}_{3}$. The mixture was filtered and the solvent carefully evaporated in vacuum. The residue was chromatographed on silica gel (Merck, Kieselgel 60, 70-230 mesh ASTM) with $\mathrm{CCl}_{4}$ as eluent unless otherwise stated.

$C p C u P B u_{3}$ and bromophenylmethane. Freshly distilled bromophenylmethane $(25 \mathrm{mmol})$ in ether $(50 \mathrm{ml})$ was added to $\mathrm{CpCuPBu}_{3}(40$ $\mathrm{mmol})$ in ether $(50 \mathrm{ml})$. Isolation and separation yielded cyclopentadienylphenylmethane as a yellowish oil $(1.8 \mathrm{~g}, 46 \%)$ consisting of $78 \%$ of the 1 -isomer and $22 \%$ of the 2 -isomer. NMR $(270 \mathrm{MHz}): \delta 7.32-7.13(5 \mathrm{H}, \mathrm{m})$ aromatic protons, $6.38(1 \mathrm{H}, \mathrm{m}), 6.23(1 \mathrm{H}, \mathrm{m}), 6.1$ and 5.97 (l $\mathrm{H}, \mathrm{m})$ olefinic protons, $3.69(2 \mathrm{H}, \mathrm{m})$ methylene protons, 2.93 and $2.81(2 \mathrm{H}, \mathrm{m})$ methylene protons in the cyclopentadienyl ring. The mixture was treated with maleic anhydride to give 1-benzylnorborn-5-ene-2,3dicarboxylic anhydride (m.p. $122-123^{\circ} \mathrm{C}$, lit. $\left.123^{\circ} \mathrm{C}\right) . .^{14}$

CpCuPBus and dibromophenylmethane. Dibromophenylmethane ${ }^{15}(10 \mathrm{mmol})$ in ether $(50 \mathrm{ml})$ was added to an ethereal solution $(50 \mathrm{ml})$ of $\mathrm{CpCuPBu}_{3}(30 \mathrm{mmol})$ at $0^{\circ} \mathrm{C}$. Isolation and separation gave dicyclopentadienylphenylmethane as a yellow oil $(0.97 \mathrm{~g}, 44 \%)$ consisting of three isomers, the 1,1 -isomer $(39 \%)$, the 1,2 -isomer $(50 \%)$, and the 2,2 -isomer (11 \%). NMR (270 MHz): $\delta 7.36-7.20(5 \mathrm{H}, \mathrm{m})$ aromatic protons, $6.43-6.28,6.20,5.99,5.88$ (6 H, m) olefinic protons, $4.91(1 \mathrm{H}, \mathrm{m})$ methine protons, $3.02,2.99,2.94$, and $2.92(4 \mathrm{H}, \mathrm{m})$ methylene protons. MS $(70 \mathrm{eV}): m / e 220(\mathrm{M}+, 100 \%), 155$ (64), 142 (19), and 77 (23). Abs. mass: Found 220.125; calc. for $\mathrm{C}_{16} \mathrm{H}_{17} 220.125$. trans-Stilbene was also isolated from the reaction $(270 \mathrm{mg}$, $30 \%$, m.p. $123^{\circ} \mathrm{C}$, lit. $\left.{ }^{16} 125^{\circ} \mathrm{C}\right)$.

$C p C u P B u_{3}$ and tribromophenylmethane. Tribromophenylmethane ${ }^{15}(5 \mathrm{mmol})$ in ether $(50 \mathrm{ml})$ was added to an ethereal solution $(200 \mathrm{ml})$ of $\mathrm{CpCuPBu}_{3}(22 \mathrm{mmol})$ at $0^{\circ} \mathrm{C}$. Isolation and separation yielded tricyclopentadienylphenylmethane as a yellow oil $(0.86 \mathrm{~g}$,
$59 \%$ ) consisting of four isomers. NMR (270 $\mathrm{MHz}): \delta 7.25-7.11(5 \mathrm{H}, \mathrm{m})$ aromatic protons, $6.48-6.26,6.18,6.15,5.97$, and $5.86(9 \mathrm{H}, \mathrm{m})$ olefinic protons, 2.97, and $2.92(6 \mathrm{H}, \mathrm{m})$ methylene protons. MS $(40 \mathrm{eV}): m / e 284(\mathrm{M}+, 18 \%)$, 219 (6), 178 (100), $115(12)$, and 91 (10). UV $\left(\mathrm{CH}_{2} \mathrm{Cl}_{2}\right): \lambda_{\max } 252 \mathrm{~nm}$ (log $\left.\varepsilon 3.69\right)$. IR (film): 3066 (s), 2900 (s), 1605 (s), 1495 (s), 1382 (s), $1360(\mathrm{~s}), 1240(\mathrm{~m}), 1190(\mathrm{~m}), 1082(\mathrm{~m}), 1040$ (s), $1015(\mathrm{~m}), 912(\mathrm{~s})$, and $795(\mathrm{~s}) \mathrm{cm}^{-1}$. The yield is highly dependent on the concentration of reactants. Thus a sixfold increase in con. centration resulted in only a $5 \%$ yield of tricyclopentadienylphenylmethane. Dibromostilbene was also isolated from the first reaction $\left(0.11 \mathrm{~g}, 13 \%\right.$, m.p. $205^{\circ} \mathrm{C}$, lit. $\left.206-208^{\circ} \mathrm{C}{ }^{17}\right)$. $\mathrm{CpCuPBu_{3 }}$ and bromodiphenylmethane, dibromodiphenylmethane, and chlorotriphenylmethane. $\mathrm{CpCuPBu}_{3}$ was reacted with bromodiphenylmethane, dibromodiphenylmethane, and chlorotriphenylmethane under the same conditions, and the products isolated as described above. In the last-named reaction we observed a crystalline product formed after a few hours, which was collected and identified as pure 1-(triphenylmethyl)cyclopentadiene. NMR (270 $\mathrm{MHz}): \delta 7.23(15 \mathrm{H}, \mathrm{m})$ aromatic protons, 6.42 , 6.33 and $6.28(3 \mathrm{H}, \mathrm{m})$ olefinic protons and 2.89 ( $2 \mathrm{H}, \mathrm{m})$ methylene protons. The isomerisation to the equilibrium mixture was followed by NMR spectroscopy (methylene protons). The rate constant for the thermal isomerisation at $22^{\circ} \mathrm{C}$ of the 1 -isomer to the 2 -isomer was determined to be $5.1 \times 10^{-6}$ and the rate constant for the reverse reaction calculated to be $1.4 \times 10^{-6} \mathrm{~s}^{-1}$ from the equilibrium constant of $3.8 .^{\circ}$

Acknowledgements. We thank Prof. Martin Nilsson for valuable discussions and suggestions, Mrs. Marianne Frantsi for skilful technical assistance and the Swedish Natural Science Research Council for financial support.

\section{REFERENCES}

1. a. Delbaere, L. T. J., McBride, D. W. and Ferguson, R. B. Acta Chrystallogr. B 26 (1970) 515; b. Cotton, F. A. and Marks, T. J. J. Am. Chem. Soc. 91 (1969) 7281.

2. See, e.g. a. Sih, C. J., Salomon, R. G., Prize, P., Sood, R. and Peruzotti, G. J. Am. Chem. Soc. 97 (1975) 857; b. Wilcox, C. F. and Whitney, G. C. J. Org. Chem. 32 (1967) 2933; c. Schmidt, C. H. Chem. Ber. 91 (1958) 28; d. Murahashi, S. I., Hino, K., Maeda, Y. and Monitani, I. Tetrahedron Lett. (1973) 3005.

3. Nilsson, M., Wahren, R. and Wennerström, O. Tetrahedron Lett. (1970) 4583.

4. Lundin, R., Moberg, C., Wahren, R. and Wennerström, O. Acta Chem. Scand. 26 (1972) 2045.

Acta Chem. Scand. B 32 (1978) No. 7 
5. Månsson, J.-E., Nilsson, M. and Wennerström, O. Acta Chem. Scand. B 31 (1977) 47.

6. Werner, H., Mattmann, G., Salzer, A. and Winkler, T. J. Organomet. Chem. 25 (1970) 461.

7. McLean, S. and Haynes, P. Tetrahedron 21 (1965) 2313, 2329.

8. a. Hutchings, M. G., Andose, J. D. and Mislow, K. J. Am. Chem. Soc. 97 (1975) 4552 and $4562 ; \mathrm{b}$. Andose, J. D. and Mislow, K. Ibid. 96 (1974) 2168; c. Gust, D. and Mislow, K. Ibid. 95 (1973) 1535; d. Finocchiaro, P., Gust, D. and Mislow, K. Ibid. 95 (1973) 8173.

9. Månson, J.-E., Olsson, T. and Wennerström, 0 . To be published.

10. Wennerström, O. and Raston, I. Acta Chem. Scand. $B 32$ (1978) 463.

11. Jacobson, I. T. Acta Chem. Scand. 21 (1967) 2235; Woodward, R. B., Fukunaga, T. and Kelly, R. C. J. Am. Chem. Soc. 86 (1964) 3164.

12. Paquette, L. A., Snow, R. A., Malhard, J. L. and Cynkowski, T. J. Am. Chem. Soc. 100 (1978) 1600.

13. Cotton, F. A. and Marks, T. J. J. $A m$. Chem. Soc. 92 (1970) 5114.

14. Alder, $K$. and Holtzrichter, H. Justus Liebigs Ann. Chem. 524 (1936) 146.

15. Heble, L. S., Nadkarni, D. R. and Wheeler, T. S. J. Chem. Soc. (1938) 1322.

16. Smith, R. H. and Andrew, D. H. J. Am. Chem. Soc. 53 (1931) 3644.

17. Bergmann, E. J. Chem. Soc. (1936) 402.

Received April 5, 1978. 